Larga evolución de un paciente diagnosticado de cáncer microcítico de pulmón con afectación del sistema nervioso central

\title{
Long-term evolution of a patient diagnosed of small lung cancer with extension to the central nervous system
}

\author{
M. Barrado Los Arcos' ${ }^{1}$ M. Rico Osés ${ }^{1}$ M. Errasti Viader ${ }^{1}$, M. Campo Vargas ${ }^{1}$, \\ M.V. Zelaya Huerta ${ }^{2}$, E. Martínez López ${ }^{1}$
}

\section{RESUMEN}

El cáncer de pulmón es la principal causa de muerte por cáncer en hombres y mujeres. Presentamos el caso de un varón diagnosticado de un carcinoma microcítico de pulmón metastásico desde el inicio, con enfermedad estable en la actualidad, cuarenta y siete meses después del diagnóstico, tras recibir diferentes modalidades de tratamiento.

Palabras clave. Cáncer microcítico de pulmón. Metástasis cerebral. Radioterapia. Pronóstico.

\begin{abstract}
Cell lung cancer is the principal cause of cancer death in men and women. We report the case of a man diagnosed with small cell lung cancer, metastatic from the outset. The disease is stable at present, fortyseven months from diagnosis, after receiving different treatment modalities.
\end{abstract}

Keywords. Small cell lung cancer. Brain metastases. Radiotherapy. Prognosis.
1. Servicio de Oncología Radioterápica. Complejo Hospitalario de Navarra. Pamplona

2. Servicio de Anatomía Patológica. Complejo Hospitalario de Navarra. Pamplona

\section{Recepción: 05-10-2015}

Aceptación provisional: 13-01-2016

Aceptación definitiva: 11-02-2016
Correspondencia:

Marta Barrado Los Arcos

C/ Mayor, 7

31320 Milagro

Navarra

Email: martabarrado@hotmail.com 


\section{INTRODUCCIÓN}

El cáncer de pulmón es la causa principal de muerte por cáncer en hombres y mujeres. Representa el 13\% de todos los diagnósticos de cáncer y el 29\% de las muertes por cáncer ${ }^{1}$.

Existen dos tipos principales de cáncer de pulmón: de células no pequeñas (CPC$\mathrm{NP})$ y de células pequeñas (CPCP). El tipo de cáncer de pulmón y el estadio de la enfermedad definen el tipo de tratamiento necesario y el pronóstico de la enfermedad, siendo generalmente de peor pronóstico el CPCP ya que la mayoría de ellos se diagnostican con enfermedad avanzada o diseminada.

\section{CASO CLÍNICO}

Presentamos el caso de un varón de 55 años con antecedentes personales de ulcus bulbar (Helicobacter pylori positivo, erradicada en 2005), tromboembolismo pulmonar en 2012 e intervenido de hernia inguinal derecha. Fumador, con un total acumulado de 20 paquetes/año.

En mayo de 2011 acudió al Servicio de Urgencias por cuadro de inestabilidad en la marcha con lateropulsión a la derecha, bradipsiquia con disnomia y vómitos. Se le realizó un TAC craneal que mostraba una lesión en hemisferio cerebeloso derecho (Fig. 1). Tras completar estudio de extensión con TAC toraco-abdominal (Fig. 2), RM craneal, broncoscopia y PET fue diagnosticado de una lesión cerebral sugestiva de metástasis en el contexto de una posible neoplasia pulmonar localizada. Se decidió realizar metastasectomía cerebral diagnóstico-terapéutica. El resultado anatomopatológico fue de carcinoma microcítico de pulmón (Fig. 3). El diagnóstico definitivo fue de CPCP T1N0M1b, estadio IV. Se administró tratamiento adyuvante con radioterapia holocraneal hasta alcanzar dosis total de 37.5 Gys que finalizó en julio de 2011 tras lo cual recibió tratamiento de quimioterapia con cisplatino -VP16.

Precisó cambio a carboplatino por emesis e insuficiencia renal recibiendo en total 4 ciclos de tratamiento con mala tolerancia clínica y hematológica finalizando en octubre de 2011. Posteriormente, en diciembre de 2011, recibió radioterapia torácica de consolidación, 45 Gys en 15 fracciones siguiendo las recomendaciones RTOG $0937^{2}$, consiguiendo mantener la estabilidad de la enfermedad en los siguientes controles.

En julio de 2012 ingresó procedente del servicio de Urgencias por pérdida de fuerza de la extremidad inferior izquierda de varios días de

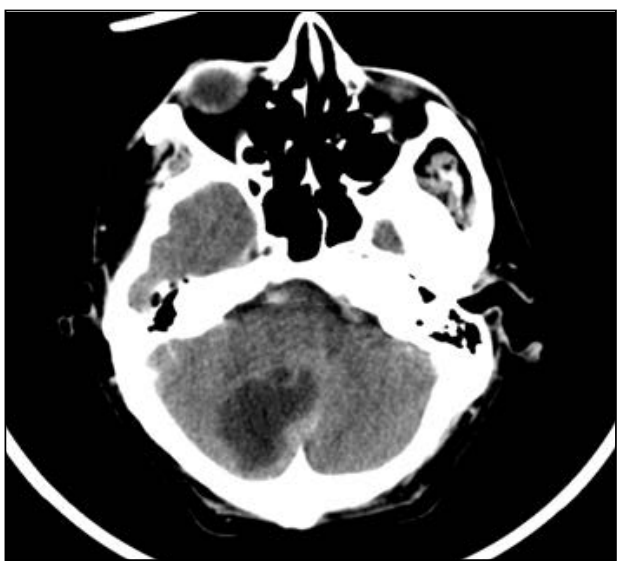

Figura 1. Lesión en hemisferio cerebeloso derecho.

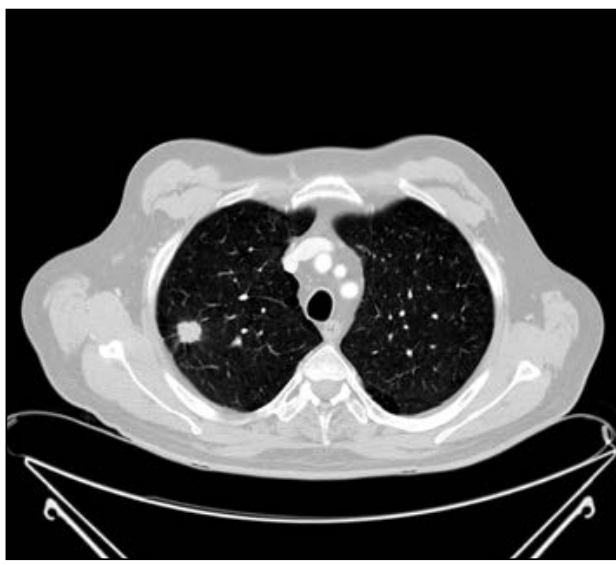

Figura 2. Lesión pulmonar sugestiva de tumoración primaria pulmonar.

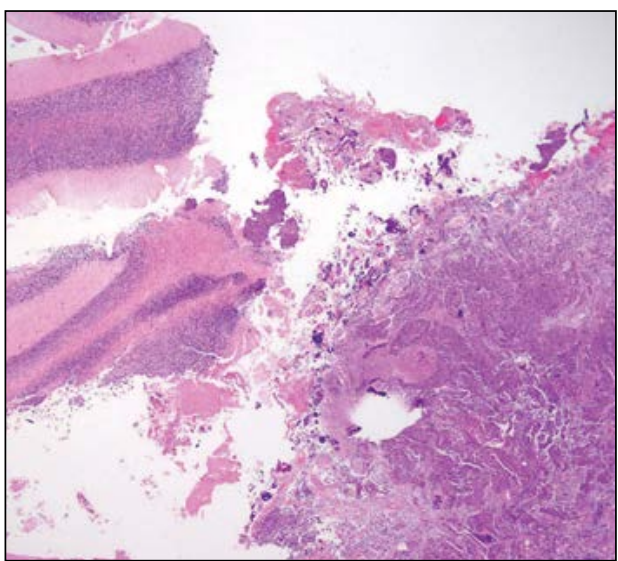

Figura 3. Carcinoma metastásico en cerebelo. Hemotoxilina-eosina. 
evolución. Se le realizó RM donde se objetivó metástasis leptomeníngeas con implante dorsal (D2-D3) que provocaba una compresión medular por lo que se inició tratamiento con radioterapia sobre niveles D1-D4 administrándose 20 Gy en 5 fracciones con excelente respuesta clínica.

Asimismo se realizó TAC toracoabdominal que mostraba adenopatía hiliar derecha de 2 cm de nueva aparición por lo que se comenzó tratamiento con carboplatino-etopósido, con respuesta parcial tras 3 ciclos y estabilización tras el $6^{\circ}$ ciclo que se administró en diciembre de 2012.

En RM de control, en junio de 2013 se visualizaron múltiples imágenes de captación puntiformes compatibles con diseminación leptomeníngea a lo largo del raquis, tanto a nivel dorsal como lumbar, encontrándose los implantes más destacables a nivel lumbar por lo que se planificó RT a ese nivel (L1-L5). Se administraron 20 Gys en 5 fracciones el mismo mes de junio de 2013.

En estudio de control mediante TAC toracoabdominal en junio de ese año se observó que la adenopatía hiliar derecha había aumentado ligeramente de tamaño respecto al TAC previo por lo que se decidió administrar radioterapia sobre la lesión hiliar con un esquema de 40 Gy en 15 fracciones, finalizando tratamiento en noviembre 2013.

Desde entonces se encuentra en revisiones, con buen estado general, vida activa e independiente para las actividades básicas de la vida diaria. En los estudios de control no hay datos de progresión de enfermedad. No se observan realces patológicos leptomeníngeos en la última RM de columna y se ha comprobado por TAC la disminución de la adenopatía hiliar derecha que en estos momentos es infracentimétrica.

\section{DISCUSIÓN}

El cáncer de pulmón es la principal causa de mortalidad por cáncer en el mundo. El CPCP corresponde al 20-25\% del total del cáncer de pulmón. Su incidencia está fuertemente relacionada con el consumo de tabaco, considerado principal factor de riesgo en la carcinogénesis de este subtipo ${ }^{3,4}$, aunque actualmente está disminuyendo precisamente por la disminución del hábito tabáquico.

El comportamiento biológico de esta enfermedad consiste en un rápido crecimiento tumoral y la diseminación metastásica precoz. El CPCP es altamente sensible a la quimioterapia y la radioterapia inicialmente aunque, en la mayoría de los casos, se vuelve resistente al tratamiento a los pocos meses presentando un alto índice de recaídas locales y a distancia.

Clásicamente se ha distinguido entre el CPCP con enfermedad limitada (CPCP-EL) y CPCP con enfermedad extendida (CPCPEE) según si la enfermedad se encuentra confinada a un hemitórax con o sin afectación hiliar, mediastínica o linfática supraclavicular ipsilateral o, por el contrario, su extensión no es abarcable por los campo de radioterapia, lo que incluye la enfermedad metastásica.

El CPCP-EE es la forma más frecuente de presentación, $60-75 \%$. Se estima que su mediana de supervivencia es de 9 meses y su tasa de curación a los 5 años es menor al $5 \%$. Sin tratamiento, la mediana de supervivencia es apenas de 1,5 meses. En el caso del CPCP-EL la mediana de supervivencia es de 12-20 meses con una supervivencia a 5 años menor del $20 \%$ y tan solo de 3 meses sin tratamiento ${ }^{5}$.

El tratamiento del CPCP-EL en pacientes seleccionados con buen estado general se basa en la administración concomitante de quimioterapia basada en etopósido y platino con radioterapia torácica y posterior radioterapia holocraneal profiláctica $(\mathrm{PCI})$, debido a la gran capacidad de generar metástasis a nivel cerebral. Si el paciente no es candidato a tratamiento concomitante se puede valorar realizar quimioterapia y radioterapia de manera secuencial o bien comenzar con un programa de cuidados paliativos.

En el caso de CPCP-EE la quimioterapia es el tratamiento inicial de elección. En aquellos casos en los que se produzca una respuesta parcial o completa tras la quimioterapia, la PCI ha demostrado que disminuye el riesgo de progresión cerebral y que aumenta la supervivencia global. Existen asímismo estudios que han mostrado que la radioterapia torácica de consolidación tras la quimioterapia puede aumentar el tiempo libre de progresión así como la supervivencia global en pacientes debidamente seleccionados. Actualmente están en marcha ensayos clínicos randomizados 
para estudiar mejor este aspecto. Todo esto puede y debe ser combinado con tratamiento paliativo de soporte ${ }^{6}$.

Diversos estudios $^{7-8}$ han analizado componentes sociales, biológicos y moleculares que permitan determinar factores asociados a mejor o peor pronóstico. En este sentido el ser joven, mujer, asiático, casado y con mayor nivel sociocultural resultan factores favorables ${ }^{9}$, pero el aumento de LDH, aumento de CEA y aumento de VEGF se han relacionado con peor pronóstico ${ }^{10}$. Sin embargo, el pronóstico individual de los pacientes es muy variable. El principal factor predictor pronóstico es la extensión de la enfermedad ${ }^{11}$, así como el número de metástasis y su localización, mostrándose una peor evolución cuando éstas se desarrollan en el sistema nervioso central o en el hígado. El tener un buen performance status al diagnóstico y no presentar pérdida ponderal, influye positivamente en la supervivencia ${ }^{12}$.

El consumo de tabaco es otro factor independiente que influye en la supervivencia del CPCP. Los pacientes fumadores tienen peor pronóstico y la continuación del hábito tabáquico durante el tratamiento influye negativamente ya que contribuye a una mayor quimiorresistencia ${ }^{13}$.

El tratamiento recibido como primera opción se ha analizado también como factor predictor de supervivencia. En el estudio retrospectivo publicado en 2012 por Gaspar y col ${ }^{14}$ sobre la evolución de la supervivencia en el CPCP a lo largo de 15 años, la administración de radioterapia torácica además de quimioterapia así como la administración de PCI se relacionaba con una mejora de la supervivencia en CPCP-EE.

En nuestro centro se administra regularmente radioterapia torácica a aquellos pacientes diagnosticados de CPCP-EE en los que se produce una buena respuesta tras la quimioterapia. Nuestros resultados presentados en el congreso de ESTRO $2012^{15}$ mostraban que este tratamiento era bien tolerado y que tras el mismo se producían pocas recaídas locales sintomáticas, resultados coincidentes con los publicados por otros grupos ${ }^{16}$.
Tras la realización de una metastasectomía cerebral, el papel de la radioterapia adyuvante ha sido ampliamente estudiado y su indicación ha sido bien establecida ${ }^{17}$ al mostrarse su rol reduciendo las recaídas intracraneales y las muertes de causa neurológica. Se ha explorado el papel de la radiocirugía o radioterapia estereotáxica fraccionada sobre el lecho quirúrgico en lugar de la administración de radioterapia holocraneal $^{18-19}$ para evitar la toxicidad derivada de ésta con resultados prometedores. Sin embargo, en nuestro caso, al tratarse de un carcinoma microcítico de pulmón, se optó por irradiar todo el cerebro ante el elevado riesgo de progresión en el SNC y estar a su vez bien establecido el papel de la PCI.

En el CPCP-EE el papel principal de la radioterapia se encuentra en la paliación de síntomas. En nuestro caso se llevó a cabo radioterapia a nivel de raquis en 2 ocasiones con este fin, con excelente respuesta clínica y sin ninguna toxicidad reseñable. La lenta evolución de la enfermedad, la buena respuesta a los tratamientos radioterápicos administrados así como la mala tolerancia a la quimioterapia inicial hizo que se planteara nuevamente la radioterapia como la opción de rescate al producirse la recidiva hiliar 18 meses tras el diagnóstico. La respuesta nuevamente fue excelente lográndose el control de la enfermedad con escasa toxicidad y postergando el uso de tratamientos sistémicos.

\section{BIBLIOGRAFÍA}

1. http://www.seom.org/es/informacion-sobre-el-cancer/info-tipos-cancer/tumorestoracios/cancer-de-pulmon?start=1\#content [consultado 25/06/2014]

2. http://www.rtog.org/ClinicalTrials/ProtocolTable/StudyDetails.aspx?study=0937 [consultado 14/04/2014]

3. González-Barcala FJ, Falagan JA, García-Prim JM, Valdes L, Carreira JM, Puga A et al. Cáncer de pulmón en el área sanitaria de Pontevedra: incidencia, presentación clínica y supervivencia. An Sist Sanit Nav 2013; 36: 217-227.

4. PolEDNAK AP. Lung cancer incidence trends by histologic type in areas of California vs. other areas in the surveillance, epidemiology and end results program. Cancer Epidemiol 2009; 33: 319-24. 
5. Christodoulou C, Skarlos DV. Treatment of small cell lung cancer. Semin Respir Crit Care Med 2005; 26: 333-341.

6. Su J, Zhu S, Liu Z, Jing S, Shen W, Li J. Analysis of limited-stage small cell lung cancer after chemoradiotherapy. Onkologie 2012; 35: 362367.

7. Spiegelmen D, Maurer LH, Ware JH, Perry MC, Chahinian AP, Comis R et al. Prognostic factors in small-cell carcinoma of the lung: an analysis of 1, 521 patients. J Clin Oncol 1989; 7: 344-354.

8. Bremnes RM, Sundstrom S, Asebu U, KaAsa S, HatLEvoll R, Aamdal S. The value of prognostic factors in small cell lung cancer: results from a randomized multicenter study with minimum 5 year follow-up. Lung Cancer 2003; 39 : 303-313.

9. Ou SH, Ziogas A, Zell JA. Prognostic factors for survival in extensive stage small cell lung cancer (ED-SCLC). The importance of smoking history, socioeconomic and marital statuses, and ethnicity. J Thorac Oncol 2009; 4: 37-43.

10. Li J, DAI CH, Chen P, Wu JN, BAO QL, QIu H et al. Survival and prognostic factors in small cell lung cancer. Med Oncol 2010; 27: 73-81.

11. Buccheri G, Ferrigno D. Prognostic factors of small cell lung cancer. Hematol Oncol Clin North Am 2004; 18: 445-460.

12. Paesmans M, Sculier JP, Lecomte J, Thiriaux J, Libert P, Sergysels R et al. Prognostic factors for patients with small cell lung carcinoma: analysis of a series of 763 patients included in 4 consecutive prospective trials with a minimum follow-up of 5 years. Cancer 2000; 89 : 523-533.
13. Martínez-García E, Irigoyen M, González-Moreno O, Corrales L, Teijeira A, Salvo E et al. Repetitive nicotine exposure leads to a more malignant and metastasis-prone phenotype of SCLC: a molecular insight into the importance of quitting smoking during treatment. Toxicol Sci 2010; 116: 467.

14. Gaspar LE, McNamara EJ, Gay EG, Putnam JB, Crawford J, Herbst RS et al. Small-cell lung cancer: prognostic factors and changing treatment over 15 years. Clin Lung Cancer 2012; 13: 115-122.

15. Rico M, Martínez E, Chicata V, Eito C, Hernández $\mathrm{B}$, Martínez $\mathrm{M}$ et al. Chest radiotherapy after chemotherapy and PCI for small cell lung cancer extensive disease. Radiother Oncol 2012; 103: 467.

16. Yee D, Butts C, Reiman A, Joy A, Smylie M, FenTON D et al. Clinical trial of post-chemotherapy consolidation thoracic radiotherapy for extensive-stage small cell lung cancer. Radiother Oncol 2012; 102: 234-238.

17. Kocher M, Soffietti R, Abacioglu U, Villa S, FAuchon F, BAUMERT BG et al. Adjuvant wholebrain radiotherapy versus observation after radiosurgery or surgical resection of one to three cerebral metastases: results of the EORTC 22952-26001 Study. J Clin Oncol 2011; 29: 134-141.

18. Aristu JJ, Ciérvide R, Guridi J, Moreno M, Arbea L, Azcona JD et al. Radioterapia estereotáctica. An Sist Sanit Navar 2009; 32: 61-71.

19. Gans JH, Raper DM, Shah AH, Bregy A, Heros D, LALLY BE et al. The role of radiosurgery to the tumor bed after resection of brain metastases. Neurosurgery 2013; 72: 317-325. 
